\title{
The Burdens of Morality: Why Act-Consequentialism Demands Too Little
}

I will understand act-consequentialism as the thesis that our moral reasons are reasons to bring about impersonally good outcomes; consequently, you morally ought not bring about a sub-optimal outcome. By evaluating these consequences impersonally, act-consequentialism faces a familiar complaint: it leaves us without personal permissions to pursue our own projects or relationships at the expense of maximising the good (Williams 1973; Wolf 1982; Scheffler 1982). In this way, act-consequentialism is said to be over-demanding sometimes. I will defend the corollary complaint that act-consequentialism is sometimes under-demanding: it fails to accommodate the fact that we have moral reasons to bear some costs ourselves, rather than pass them off onto others. Let us call these costs "burdens." To press this objection, I will give three examples of burdens.

For our first type of a burden, consider our moral reasons to internalise the costs of our beneficence. Consider Sonia who decides to climb Mount Kilimanjaro to raise money for the Special Olympics. This fund-raising appeals to her, as she loves achievement and adventure. But the training and the hike will be gruelling. Let us stipulate that the personal rewards of the trip perfectly compensate her for these hardships. Sonia's choice concerns how to finance the trip and raise money for charity. While she is reasonably well-off, her high-flyer friends are considerably better-off. Consequently, they get smaller marginal returns from their money than Sonia gets from hers. So Sonia would bring about the impersonally best outcome by relying entirely on their donations. We may suppose her friends are so indulgent of her that they would be willing to donate even if she did not donate herself. Even so, it would be wrong of Sonia to rely entirely on her friends for raising money. This would mean she has paid no net cost herself, given that the benefits of the trip perfectly balance its hardships. But since it 
is Sonia's project, she has moral reasons to bear some of these costs herself, even though allocating them elsewhere would bring about the most good.

For our second type of burden, let us turn to leadership. Consider a case where one individual has greater responsibility for a project than others have. Suppose you are a volunteer in charge of a group made up of four other volunteers. Together, you five are cleaning up the riverbank, and you have to decide who is going to clean up which stretch of the bank. Four areas are in a reasonably bad state. The fifth is just disgusting. Let us stipulate that each member of the team would find cleaning each part of the bank exactly as unpleasant. So how shall you divvy up the tasks? You could draw straws, giving everyone an equal chance of being left with the unpleasantness. But as the leader of the group, you could simply take on the dirtiest bit of the riverbank yourself. Perhaps, taking this option is not on incumbent on you, and you would be within your rights to go with the lottery. But all the same, you have a moral reason to take on the burden yourself, simply because you are the leader of the group.

As described, this case is perhaps not conclusive. An act-consequentialist might respond that there is an impersonal case for an agent to bear the burdens of leadership: by doing so, she sets an example that could have indirect consequences on other people's behaviour. In particular, she could build trust and respect among the group by showing that she is committed to the cause, and not exploiting their efforts. In the riverbank case, perhaps your selfless mucking around in the filth has this effect on the rest of the team, and consequently, the bank ends up much cleaner. ${ }^{1}$

This is a fair enough response. I suggest we partly address it by stipulating that the members of the team know and trust you well. But this is not yet an adequate way of addressing the response because even with this stipulation, we may still expect that your

\footnotetext{
${ }^{1}$ Thanks to an anonymous reviewer for suggesting that I address this response.
} 
leading by example will motivate the other team members. So to address the response fully, we should ask why it would be demoralising for other team members to see a follower do more work than a leader, or why it would motivate these team members to see their leader taking on more than her fair share. I suggest the reason is that they believe that leaders have special moral reasons to bear these burdens. We should join them in thinking this.

Third, burdens can arise when someone arrives first to a scene where action is called for. After a relaxing weekend, you come into the office only to find an abandoned teacup in the sink. You feel a pang of indignation: who has left this here over the weekend! Alas, this negligence is common-place, and you know that the person responsible for the cup will never wash it up. Instead, the cup will sit there dirty until a kind-hearted soul takes it upon themselves to clean it. So you can leave the cup for someone else to wash, knowing that eventually it will be done. Or you can wash up the cup yourself. We may suppose that considerations of the greater good do not tell one way or the other. All the same, if all else is equal, then you have a moral reason to shoulder this burden yourself. Shirking it by thinking, "Well that can be someone else's problem," would be moral laziness. If you came back later to find Maureen giving the offending cup a good scrub, I suspect your cheeks would be tinged with shame.

I do not suppose these three examples of burdens exhaust all the types of burdens there are. But these examples will do to introduce the phenomenon. Given the healthy pluralism in the moral philosophy community, I would expect readers to vary with regard to their intuitions about cases. Consequently, some readers may not agree with my claims about these agents' moral reasons for action. Alternatively, some readers may agree that the agents have these reasons, but offer explanations for these reasons that differ from my explanation. Still, by offering a variety of different types of case, I hope that for at least one case, nearly 
everyone will share the intuition that the agent in question has a moral reason to shoulder a burden.

Should we classify these reasons along with our reasons not to violate paradigmatic deontological constraints, such as constraints against lying or killing (Scheffler 1982; Nagel 1986: 164-188; Kagan 1990; McMahon 1991)? My sympathies are with distinguishing constraints from burdens. ${ }^{2}$ But since little of substance hangs on this taxonomical question, I will leave it open here. Instead, I wish to focus on pressing the challenge that burdens present for act-consequentialism as a theory of our moral reasons, by looking for the rationale for our reasons to shoulder burdens.

We might be tempted to think that these are reasons of fairness. For example, it may seem unfair of Sonia to rely wholly on her friends' donations; these costs should be distributed more evenly. But appealing to fairness in a distribution will not give us a general account of burdens. This is because burdens arise in cases where there is symmetry between the agent and the others who could bear the costs. The dirty teacup case is a paradigm of this type of case. From an impersonal point of view, it is just as fair for the burden to be the agent's or someone else's: both situations involve one person working while the others do not. Moreover, it would be silly to say that an agent would be unfair to herself, were she to choose herself as the burden-bearer. Correspondingly, it would not be unfair to another to pick her as the burden-bearer. What is going on here is not that the agent has general moral

\footnotetext{
${ }^{2}$ My reasons are twofold. First, if we assimilate burdens and constraints, then we lose the connection between constraints and complaints. A victim of lying has a special complaint against the deceiver. But Maureen would have no similar complaint that she has to clean the teacup rather than you, since you and she are symmetrically placed. Second, the rationale that I go on to defend appeals to the ethical significance of the perspectival features of our agency. This contrasts with the most plausible rationales for why it is wrong to kill or lie, which I take to be either victim-centred rationales (e.g. Kamm 1989) or relationship-centred rationales (e.g. Darwall 2006). Thanks to an anonymous reviewer for highlighting the latter type of rationales.
} 
reasons to ensure that the costs are distributed in as fair a manner as possible. Rather, she has special moral reasons to bear the cost herself, and this is simply because (e.g.) she is first to the scene.

Instead, I suggest that the case for shouldering burdens appeals to how our moral agency interacts with others' agency in group projects. Take the case of the riverbank cleaning. A virtue in a group's leader is a willingness to take on special costs herself. This is because a good leader sees her own individual agency as subordinated to a broader exercise of group agency. But being a designated leader is not the only way in which one can relate to a group. Sonia has a special position simply as someone who has initiated a fund-raising project. As initiator, she has special reasons to bear costs involved with the project. But the office-mug case brings out that one need not occupy a higher place in a group hierarchy. Instead, you can have moral reasons to bear costs simply because someone in the group must do so, and otherwise arbitrary reasons make you salient as the arm of the community. We might generalise this rationale, by saying that part of what it is to be a good moral team-player is to see oneself as a moral agent keen to look for opportunities to commit one's abilities and efforts to a shared moral cause. By contrast, a bad moral team-player looks for opportunities to hang back as a mere onlooker, and leave the group's work to others.

This brings us to why act-consequentialism faces a problem with burdens. As a fully impersonal theory, act-consequentialism gives each agent no special concern with her own agency. From an impersonal point of view, each agent's contributions are on a par with others' contributions. When colleagues stand in symmetrical relations to each other, the universe has no concern with who bears which costs per se. All that matters is how much good is produced, and nothing hangs on which means is used for this end. Not so with us, since each of us always occupies a particular perspective. From this perspective, each of us is permitted to have personal interests besides morality. But we also are subject to special moral 
demands. By ignoring these facts about agency, it is not only the case that actconsequentialism sometimes demands too much of us. In addition, act-consequentialism sometimes demands too little.

\section{References}

Darwall, S. 2006. The Second Person Standpoint: Morality, Respect, and Accountability. Cambridge MA: Harvard University Press

Kagan, S. 1989. The Limits of Morality. Oxford: Clarendon Press

Kamm, F. 1989. Harming some to save others. Philosophical Studies 57(3): 227-260.

McMahon, C. 1991. The paradox of deontology. Philosophy and Public Affairs 20(4): 350377

Nagel, T. 1986. The View From Nowhere. Oxford: Oxford University Press

Scheffler, S. 1982. The Rejection of Consequentialism. Oxford: Clarendon Press

Williams, B., 1973. "A Critique of Utilitarianism" in Utilitarianism: For and Against, by J.J.C. Smart and B. Williams. Cambridge: Cambridge University Press, pp. 77-150

Wolf, S. 1982. Moral saints. The Journal of Philosophy 79(8): 419-439 